\title{
INTERPRETATION OF EXPERIMENTS IN PAUL TRAP
}

\author{
J. DEMBCZYŃSKI, E. STACHOWSKA, J. RUCZKOWSKI, A. STACHOWSKA \\ Chair of Atomic Physics, Poznań University of Technology \\ Piotrowo 3, 60-965 Poznań, Poland \\ e-mail: $\quad$ dembczyn@phys.put.poznan.pl
}

\begin{abstract}
Chosen aspects of laser spectroscopy in a Paul trap have been discussed. On the example of interpretation of the experimental results for europium ion the advantages of the use of semiempirical atomic structure calculations have been proved. For the calculations a computer package, prepared and adopted by ourselves to be used on vector computers, has been applied.
\end{abstract}

\section{I N T RO D UCTION}

Recently hyperfine structure $(h f s)$, which is a result of the electromagnetic interaction of electronic shells with a non-spherical nucleus, has been investigated intensively using of laser spectroscopy methods. The typical accuracy of $h f s$-splitting determination by means of laser spectroscopy on an atomic beam is in a range of $\mathrm{MHz}$ and in the case of the most precise measurements using laser-rf double resonance in a Paul trap experimental error may be less than a few Hz. Unfortunately, in order to interpret the results of the measurements for complex atoms very complicated and time consuming theoretical studies und calculations are necessary. A general purpose of our work is to reach in our calculations an accuracy comparable with the accuracy of the experiments. This requires further profound studies on the atomic structure theory as well as elaboration of new calculation methods. In this work we present our semi-empirical method of fine- and hyperfine structure analysis, including a special computer package prepared for calculations with the use of vector computers. Some results of the calculations obtained for europium ion $\mathrm{Eu}^{+}$are given.

\section{EXPERIMENTS IN A PAUL TRAP}

After pioneering work of Dehmelt and Toschek [1,2] on spectroscopy of single Ba ions it became clear that spectroscopy of ions stored in a Paul trap will be an important tool for high resolution studies or new frequency standards. The Paul trap uses DC and AC electric fields to create a potential well in which the ions are trapped. It consists of a ring electrode and two end caps, all of them being hyperboloids of having hyperbolic revolution as shown in Fig. 1. Inside the trap the potential is given by

$$
V(r, z)=\frac{V}{2 r_{0}^{2}}\left(r^{2}-2 z^{2}\right)
$$

where cylindrical coordinates are used with origin at the centre of the trap, $r_{0}$ is the radius of the ring electrode at the waist and $z_{0}$ is the distance of each of the end caps from the origin. This equation applies for the case 


$$
r_{0}=\sqrt{2 z_{0}} .
$$

If $V$ is a DC potential, the centre of the trap is a potential nodal point and an ion is expelled from the region. However, if $V$ contains an oscillating component such as

$$
V=U_{1}+V_{1} \cos (\Omega t),
$$

the centre of the trap becomes a region of minimum potential energy and, under certain conditions, positive and negative ions may be trapped there.

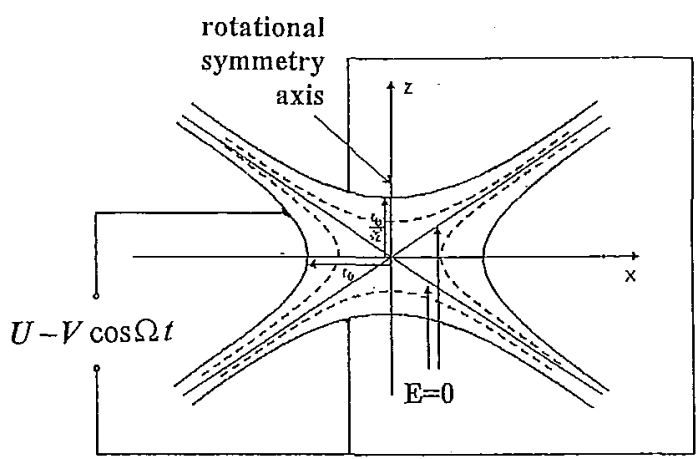

Fig. 1. Electrode structure of a Paul trap

The trapped ions have a high kinetic energy rendering them useless for many applications, such as precision spectroscopy; therefore the ions have to be cooled. This is done by laser light. The laser frequency $v_{L}$, is tuned below the resonance frequency $v_{R}$, so that the energy of the photon is not sufficient to excite the atom. However, the ion can extract the missing energy from its own motion and thus reduce its kinetic energy. In other words, for ions with resonance frequency $v_{R}$ moving towards the source of laser light at velocity $\mathrm{V}$ the incident radiation is Doppler shifted into resonance so that

$$
v_{R}=v_{L}(1+v / c)
$$

The energy absoiption process is accompanied by the transfer of momentum $\Delta p=h v / c$ from the absorbed photon to the ion under consideration. Since emission may take place in any direction with the same probability, the net effect is to change the momentum of absorbing atoms by the quantity $\mathrm{M} \Delta \mathrm{V}=h v / c$. This results in a reduction of the kinetic energy of the ion, which means a decrease of temperature. The cooling of the ions by the laser light can be effected down to the socalled Dicke limit [3] which means that the residual motion amplitude of an ion is much smaller than the wavelength of the radiation used. In this limit the first-order Doppler broadening disappears and - since the temperature is in the range of $\mu K$ - also the second-order Doppler effect is excluded. Thus, the ultimate resolution is determined by the natural linewidth of the investigated transition, or - in other words - the accuracy is only limited by the Heisenberg uncertainty principle $\Delta E \approx \hbar / \Delta t$, or in an equivalent form $\Delta v \approx 1 / \Delta t$, where $\Delta t$ is the lifetime of the excited electronic level involved. Therefore only the optical transitions from an long-lived electronic 
levels can be candidates for optical atomic frequency standards. Without exception, all ions which have been under consideration so far, have an electronic level scheme of the type shown in Fig. 2. The ground state is ${ }^{2} S_{l / 2}$, connected by electric dipole transitions to ${ }^{2} P$ levels with lifetimes of the order of $10^{-6} \mathrm{~s}$, which may partly decay into low-lying ${ }^{2} D$ states. The ${ }^{2} D$ states decay through electric quadrupole radiation into the ground state and their lifetimes exceed in some cases several hundred milliseconds. The transition ${ }^{2} S \rightarrow{ }^{2} P$ is used as a pumping and laser cooling transition while the transition ${ }^{2} D \rightarrow{ }^{2} S$ is the candidate for the optical atomic frequency standard. Moreover, the general level scheme given in the Fig. 2 offers possibilities for precise reference frequencies in three different spectral regions: the ground-state hyperfine magnetic dipole transitions in odd isotopes in the microwave domain at around $10^{10} \mathrm{~Hz}$, the optical electric quadrupole transition ${ }^{2} S \rightarrow{ }^{2} \mathrm{D}$ transition at $10^{14} \mathrm{~Hz}$, and the infrared magnetic dipole fine-structure transition ${ }^{2} D_{3 / 2} \rightarrow{ }^{2} D_{5 / 2}$ at about $10^{12} \mathrm{~Hz}$.

Fig. 2. Partial energy level diagram for $\mathrm{Ba}^{+}$

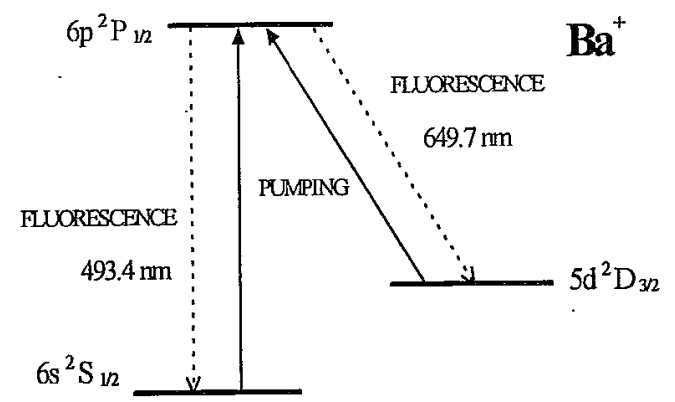

For many applications in atomic spectroscopy, however, such a high precision is generally not required, since the calculations in atomic physics do not reach the same level of accuracy. In such cases large ion clouds may be more advantageous than single ions since they offer much higher signal-to-noise ratios. The ions then, of course, will move in regions of high electric fields and Doppler effect may limit the spectral resolution. Fortunately, for hyperfine measurements in the radiofrequency or microwave domain only the second-order Doppler effect appears, since even for large ion clouds of several $\mathrm{mm}$ diameter the ion oscillation amplitude is generally smaller then the wavelength of the radiation. In this case, as shown by Dicke [3], the first-order Doppler effect shows up in sidebands at the ion oscillation frequencies, symmetrically around the unshifted and unbroadened central carrier. Second-order Doppler shifts, which limit the precision in these cases, are of the order of $10^{-11}$ and are up to now negligible for calculable effects in atomic physics.

\section{STUDIES OF THE ATOMIC STRUCTURE}

Systematic investigations of atomic structure presented in this work are carried out in accordance with the scheme given in Fig. 3. Characteristic feature of the present investigation is that experimental and theoretical studies are performed simultaneously. It makes atomic the structure analysis very effective. As an example of the structure of complex atoms, the atomic structure of $\mathrm{Eu}^{+}$has been presented in Fig. 4. In the singly ionised europium atom 8 electrons occupy open shells and can create many electronic configurations. Centers of gravity 3 configurations: 


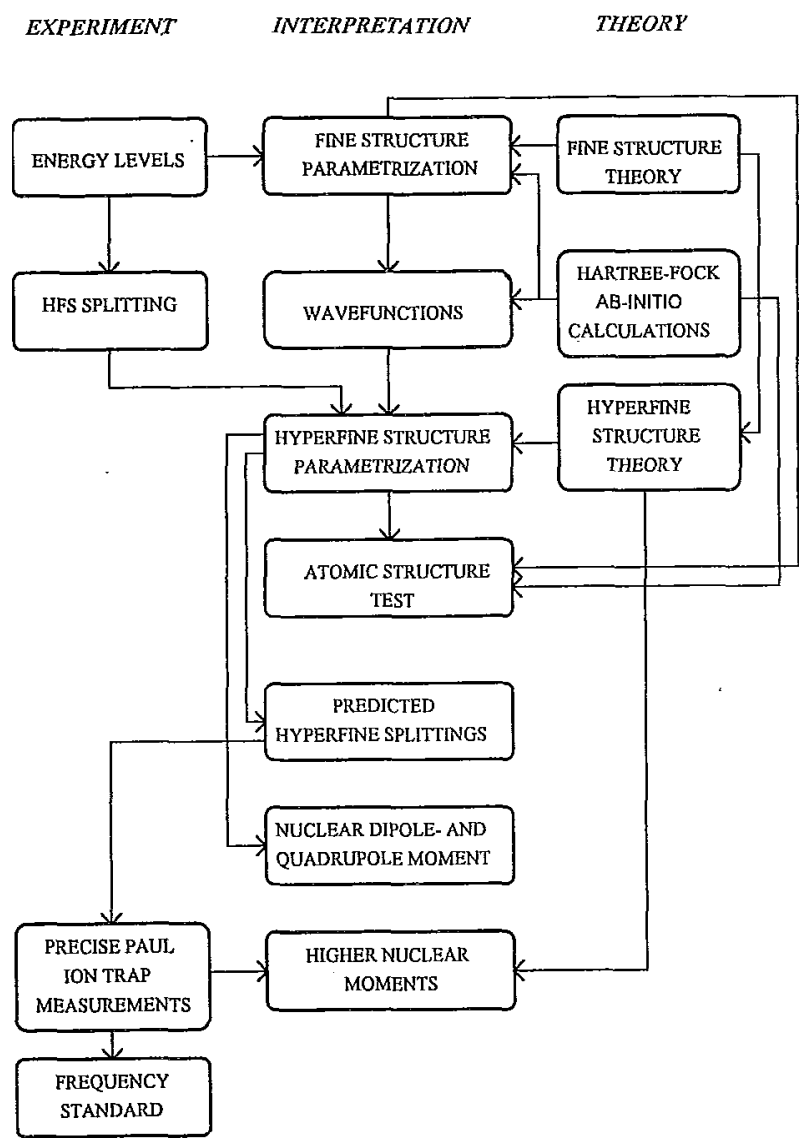

Fig. 3. Scheme of the atomic structure investigation

$4 f^{7} 6 \mathrm{~s}, 4 f^{7} 5 \mathrm{~d}$ and $4 f^{7} 6 p$ have been marked in Fig. 4 . Term structure, fine structure splittings as well as hyperfine structure splittings, have been shown in order to illustrate relative magnitudes of different interactions in atomic structure. Mutual Coulomb repulsion of electrons leads to term structure, spin-orbit interaction causes fine structure splittings. Due to the interaction of the nuclear moments with the field in the place of nucleus, originating from orbital and spin angular momenta of electrons, the hyperfine structure splittings one observed. The hyperfine structure splittings of the levels of $\mathrm{Eu}^{+}$do not exceed $10^{9} \mathrm{~Hz}$ whereas the whole atomic structure is six orders of magnitude greater and is of the order of $\sim 10^{15} \mathrm{~Hz}$. The hyperfine splitting of the ground state or metastable states can be measured with an accuracy of about $1 \mathrm{~Hz}$. To these splittings contribute not only the interactions of the nucleus with the electrons belonging to the ground configuration but also the interactions involving the electrons belonging to many excited configurations, which lie in the above mentioned energy region of $10^{15} \mathrm{~Hz}$. Even though in eigenvectors compositions of the ground state $4 f^{7}\left({ }^{8} S_{7 / 2}\right) 6 s^{9} S_{4}$ and of the lowest-lying metastable state $4 f^{7}\left({ }^{8} S_{7 / 2}\right) 6 s^{7} S_{3}$ dominate the vectors of one configuration only $(99 \%)$, the relative accuracy of the 


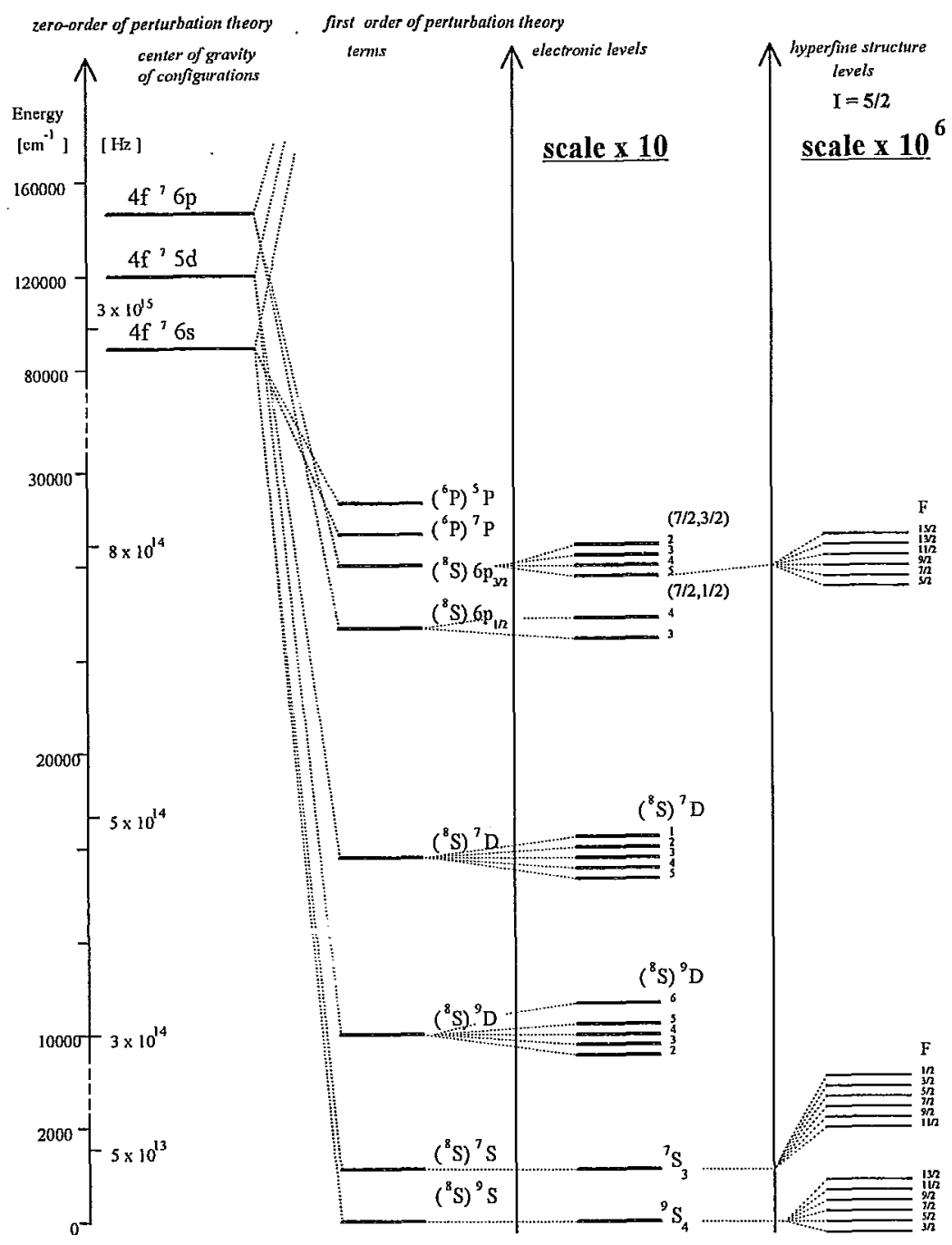

Fig. 4. Partial energy level diagram for $\mathrm{Eu}^{+}$

routine calculations in one-configuration approximation can not be better than $10^{-2} \div 10^{-3}$. Such a precision is a very poor one in comparison with the present experimental accuracy of $10^{-8} \div 10^{-10}$. Thus in order to improve the calculations the effects of interactions with excited configurations have to be taken into account. Interacting configurations are presented schematically in Fig. 4. As a result of electrostatic interaction between configurations with the same parity, which differ in quantum numbers of two electrons at most, the electronic states of the interacting configurations "mix" with one another. In other words the quantum numbers $n$ and $l$ are no more "good quantum numbers". Configuration mixing effects can be also caused due to 
Table I. Experimental and calculated energy of odd parity levels of Eu ion (up to $\sim 40000 \mathrm{~cm}^{-1}$ )

\begin{tabular}{|c|c|c|c|c|c|c|c|c|c|c|}
\hline Designation & $\begin{array}{l}\text { Observed } \\
\left(\mathrm{cm}^{-1}\right)\end{array}$ & $\begin{array}{c}\text { Calculated } \\
\left(\mathrm{cm}^{-1}\right)\end{array}$ & $\begin{array}{c}\Delta E \\
\left(\mathrm{~cm}^{-1}\right)\end{array}$ & $\begin{array}{c}\text { First component } \\
(\%)\end{array}$ & & $\begin{array}{l}\text { Second component } \\
(\%)\end{array}$ & & Obs. $g_{J}$ & Calc. $g_{J}$ & $\Delta g_{J}$ \\
\hline$J=0$ & & $\begin{array}{l}38847 \\
42505\end{array}$ & & $\begin{array}{l}56.65 \\
48.36\end{array}$ & $\begin{array}{l}{ }^{7} \mathrm{~F},{ }^{3} \mathrm{P} ;{ }^{9} \mathrm{G} \\
{ }^{7} \mathrm{~F},{ }^{3} \mathrm{D} ;{ }^{9} \mathrm{G}\end{array}$ & $\begin{array}{l}29.95 \\
11.00\end{array}$ & $\begin{array}{l}{ }^{7} \mathrm{~F},{ }^{3} \mathrm{P} ;{ }^{9} \mathrm{G} \\
{ }^{7} \mathrm{~F},{ }^{3} \mathrm{P} ;{ }^{9} \mathrm{G}\end{array}$ & & . & \\
\hline $\begin{array}{r}J=1 \\
4 f^{7} 5 d^{7} \mathrm{D}\end{array}$ & 17324.66 & $\begin{array}{l}17357 \\
36993 \\
38754 \\
39211\end{array}$ & -32 & $\begin{array}{l}95.54 \\
84.92 \\
18.03 \\
38.04\end{array}$ & $\begin{array}{l}{ }^{8} \mathrm{~S} ;{ }^{7} \mathrm{~d} \\
{ }^{6} \mathrm{P} ;{ }^{5} \mathrm{P} \\
{ }^{7} \mathrm{~F} ;{ }^{3} \mathrm{D} ;{ }^{9} \mathrm{H} \\
{ }^{7} \mathrm{~F},{ }^{3} \mathrm{P} ;{ }^{9} \mathrm{G}\end{array}$ & $\begin{array}{r}1.24 \\
5.51 \\
17.39 \\
18.94\end{array}$ & $\begin{array}{l}{ }^{6} \mathrm{P} ;{ }^{5} \mathrm{P} \\
{ }^{7} \mathrm{~F} ;{ }^{3} \mathrm{~F} ;{ }^{5} \mathrm{P} \\
{ }^{7} \mathrm{~F} ;{ }^{3} \mathrm{P} ;{ }^{9} \mathrm{G} \\
{ }^{7} \mathrm{~F},{ }^{3} \mathrm{P} ;{ }^{9} \mathrm{G}\end{array}$ & $\begin{array}{l}2.994 \\
2.471 \\
0.057 \\
0.741\end{array}$ & 2.972 & -0.022 \\
\hline $\begin{array}{r}J=2 \\
4 f^{7} 5 d^{9} \mathrm{D} \\
4 f^{7} 5 d^{7} \mathrm{D}\end{array}$ & $\begin{array}{r}9923.00 \\
17247.67\end{array}$ & $\begin{array}{r}9946 \\
17275 \\
35713 \\
36645 \\
37228 \\
39100 \\
39805\end{array}$ & $\begin{array}{l}-23 \\
-27\end{array}$ & $\begin{array}{l}96.75 \\
95.09 \\
83.52 \\
79.55 \\
86.31 \\
23.58 \\
28.77\end{array}$ & $\begin{array}{l}{ }^{8} \mathrm{~S} ;{ }^{9} \mathrm{D} \\
{ }^{8} \mathrm{~S} ;{ }^{7} \mathrm{D} \\
{ }^{6} \mathrm{P} ;{ }^{7} \mathrm{P} \\
{ }^{6} \mathrm{P} ;{ }^{5} \mathrm{P} \\
{ }^{7} \mathrm{~F} ;{ }^{3} \mathrm{~F} ;{ }^{9} \mathrm{I} \\
{ }^{7} \mathrm{~F} ;{ }^{3} \mathrm{P} ;{ }^{9} \mathrm{G} \\
{ }^{7} \mathrm{~F} ;{ }^{3} \mathrm{P} ;{ }^{9} \mathrm{G}\end{array}$ & $\begin{array}{r}1.27 \\
0.82 \\
5.51 \\
7.92 \\
6.59 \\
16.77 \\
22.31\end{array}$ & $\begin{array}{l}{ }^{6} \mathrm{P} ;{ }^{7} \mathrm{P} \\
{ }^{6} \mathrm{P} ;{ }^{5} \mathrm{P} \\
{ }^{6} \mathrm{D} ;{ }^{7} \mathrm{D} \\
{ }^{6} \mathrm{D} ;{ }^{5} \mathrm{D} \\
{ }^{7} \mathrm{~F},{ }^{1} \mathrm{D} ;{ }^{7} \mathrm{H} \\
{ }^{7} \mathrm{~F} ;{ }^{3} \mathrm{P} ;{ }^{9} \mathrm{G} \\
{ }^{7} \mathrm{~F},{ }^{3} \mathrm{D} ;{ }^{9} \mathrm{H}\end{array}$ & $\begin{array}{l}2.660 \\
2.000\end{array}$ & $\begin{array}{r}2.641 \\
1.981 \\
2.304 \\
1.820 \\
-0.286 \\
1.113 \\
1.133 \\
\end{array}$ & $\begin{array}{l}-0.019 \\
-0.019\end{array}$ \\
\hline $\begin{array}{c}J=3 \\
4 f^{7} 6 s^{7} \mathrm{~S} \\
4 f^{7} 5 d^{9} \mathrm{D} \\
4 f^{7} 5 d^{7} \mathrm{D}\end{array}$ & $\begin{array}{r}1669.21 \\
10081.65 \\
17140.87\end{array}$ & $\begin{array}{r}1669 \\
10098 \\
17154 \\
35430 \\
37129 \\
37806 \\
38737 \\
39666 \\
40588\end{array}$ & $\begin{array}{r}0 \\
-16 \\
-13\end{array}$ & $\begin{array}{l}94.46 \\
96.20 \\
94.54 \\
83.18 \\
78.43 \\
86.50 \\
90.76 \\
26.64 \\
34.93\end{array}$ & $\begin{array}{l}{ }^{8} \mathrm{~S} ;{ }^{7} \mathrm{~S} \\
{ }^{8} \mathrm{~S} ;{ }^{9} \mathrm{D} \\
{ }^{8} \mathrm{~S} ;{ }^{7} \mathrm{D} \\
{ }^{6} \mathrm{P} ;{ }^{7} \mathrm{P} \\
{ }^{6} \mathrm{P} ;{ }^{5} \mathrm{P} \\
{ }^{7} \mathrm{~F} ;{ }^{3} \mathrm{~F} ;{ }^{9} \mathrm{I} \\
{ }^{6} \mathrm{I} ;{ }^{7} \mathrm{I} \\
{ }^{7} \mathrm{~F} ;{ }^{3} \mathrm{P} ;{ }^{9} \mathrm{G} \\
{ }^{7} \mathrm{~F},{ }^{3} \mathrm{D} ;{ }^{9} \mathrm{H}\end{array}$ & $\begin{array}{r}2.23 \\
0.94 \\
1.08 \\
8.45 \\
9.93 \\
6.14 \\
5.08 \\
20.97 \\
20.98\end{array}$ & $\begin{array}{l}{ }^{7} \mathrm{~F} ;{ }^{1} \mathrm{~F} ;{ }^{7} \mathrm{~S} \\
{ }^{8} \mathrm{~S} ;{ }^{7} \mathrm{D} \\
{ }^{6} \mathrm{P} ;{ }^{5} \mathrm{D} \\
{ }^{6} \mathrm{D} ;{ }^{7} \mathrm{D} \\
{ }^{6} \mathrm{D} ;{ }^{5} \mathrm{D} \\
{ }^{7} \mathrm{~F} ;{ }^{1} \mathrm{D} ;{ }^{7} \mathrm{H} \\
{ }^{7} \mathrm{~F} ;{ }^{3} \mathrm{~F} ;{ }^{7} \mathrm{I} \\
{ }^{7} \mathrm{~F} ;{ }^{3} \mathrm{P} ;{ }^{9} \mathrm{G} \\
{ }^{7} \mathrm{~F},{ }^{3} \mathrm{P} ;{ }^{9} \mathrm{G}\end{array}$ & $\begin{array}{l}1.997 \\
2.078 \\
1.751\end{array}$ & $\begin{array}{l}1.981 \\
2.062 \\
1.728 \\
1.901 \\
1.655 \\
0.604 \\
0.250 \\
1.332 \\
1.248\end{array}$ & $\begin{array}{l}-0.016 \\
-0.016 \\
-0.023\end{array}$ \\
\hline
\end{tabular}




\begin{tabular}{|c|c|c|c|c|c|c|c|c|c|c|}
\hline $\begin{array}{c}J=4 \\
4 f^{7} 6 s^{9} \mathrm{~S} \\
4 f^{7} 5 d^{9} \mathrm{D} \\
4 f^{7} 5 d^{7} \mathrm{D}\end{array}$ & $\begin{array}{r}0.00 \\
10312.82 \\
17004.06\end{array}$ & $\begin{array}{r}0 \\
10318 \\
16995 \\
35960 \\
38545 \\
38766 \\
40076 \\
40421 \\
41468\end{array}$ & $\begin{array}{r}0 \\
-5 \\
13\end{array}$ & $\begin{array}{l}94.81 \\
95.69 \\
94.15 \\
80.57 \\
79.73 \\
84.44 \\
92.12 \\
28.34 \\
52.56\end{array}$ & $\begin{array}{l}{ }^{8} \mathrm{~S} ;{ }^{9} \mathrm{~S} \\
{ }^{8} \mathrm{~S} ;{ }^{9} \mathrm{D} \\
{ }^{8} \mathrm{~S} ;{ }^{7} \mathrm{D} \\
{ }^{6} \mathrm{P} ;{ }^{7} \mathrm{P} \\
{ }^{7} \mathrm{~F} ;{ }^{3} \mathrm{~F} ;{ }^{9} \mathrm{I} \\
{ }^{6} \mathrm{I} ;{ }^{7} \mathrm{I} \\
{ }^{6} \mathrm{I} ;{ }^{5} \mathrm{I} \\
{ }^{7} \mathrm{~F} ;{ }^{3} \mathrm{P} ;{ }^{9} \mathrm{G} \\
{ }^{7} \mathrm{~F} ;{ }^{3} \mathrm{D} ;{ }^{9} \mathrm{H}\end{array}$ & $\begin{array}{r}3.45 \\
1.44 \\
1.61 \\
9.77 \\
7.08 \\
7.89 \\
4.61 \\
24.67 \\
16.35\end{array}$ & $\begin{array}{l}{ }^{7} \mathrm{~F} ;{ }^{3} \mathrm{~F} ;{ }^{9} \mathrm{~S} \\
{ }^{8} \mathrm{~S} ;{ }^{7} \mathrm{D} \\
{ }^{8} \mathrm{~S} ;{ }^{9} \mathrm{D} \\
{ }^{6} \mathrm{D} ;{ }^{7} \mathrm{D} \\
{ }^{6} \mathrm{I} ;{ }^{7} \mathrm{I} \\
{ }^{7} \mathrm{~F} ;{ }^{3} \mathrm{~F} ;{ }^{9} \mathrm{I} \\
{ }^{7} \mathrm{~F} ;{ }^{3} \mathrm{~F} ;{ }^{5} \mathrm{I} \\
{ }^{7} \mathrm{~F} ;{ }^{3} \mathrm{P} ;{ }^{9} \mathrm{G} \\
{ }^{7} \mathrm{~F} ;{ }^{3} \mathrm{~F} ;{ }^{9} \mathrm{H}\end{array}$ & $\begin{array}{l}1.998 \\
1.845 \\
1.651\end{array}$ & $\begin{array}{l}1.984 \\
1.842 \\
1.628 \\
1.743 \\
0.943 \\
0.768 \\
0.600 \\
1.409 \\
1.283\end{array}$ & $\begin{array}{l}-0.014 \\
-0.003 \\
-0.023\end{array}$ \\
\hline $\begin{array}{r}J=5 \\
4 f^{7} 5 d^{9} \mathrm{D} \\
4 f^{7} 5 d^{7} \mathrm{D}\end{array}$ & $\begin{array}{l}10643.48 \\
16860.72\end{array}$ & $\begin{array}{l}10633 \\
16798 \\
38758 \\
39481 \\
40067 \\
41337 \\
\end{array}$ & $\begin{array}{l}11 \\
62\end{array}$ & $\begin{array}{l}95.67 \\
94.49 \\
91.05 \\
87.94 \\
92.19 \\
29.13\end{array}$ & $\begin{array}{l}{ }^{8} \mathrm{~S} ;{ }^{9} \mathrm{D} \\
{ }^{8} \mathrm{~S} ;{ }^{7} \mathrm{D} \\
{ }^{6} \mathrm{I} ;{ }^{7} \mathrm{I} \\
{ }^{7} \mathrm{~F} ;{ }^{3} \mathrm{~F} ;{ }^{9} \mathrm{I} \\
{ }^{6} \mathrm{I} ;{ }^{5} \mathrm{I} \\
{ }^{7} \mathrm{~F} ;{ }^{3} \mathrm{P} ;{ }^{9} \mathrm{G}\end{array}$ & $\begin{array}{r}1.50 \\
1.68 \\
4.18 \\
4.61 \\
4.35 \\
27.93\end{array}$ & $\begin{array}{l}{ }^{8} \mathrm{~S} ;{ }^{7} \mathrm{D} \\
{ }^{8} \mathrm{~S} ;{ }^{9} \mathrm{D} \\
{ }^{7} \mathrm{~F},{ }^{3} \mathrm{~F} ;{ }^{7} \mathrm{I} \\
{ }^{7} \mathrm{~F},{ }^{1} \mathrm{D} ;{ }^{7} \mathrm{H} \\
{ }^{7} \mathrm{~F},{ }^{3} \mathrm{~F} ;{ }^{5} \mathrm{I} \\
{ }^{7} \mathrm{~F},{ }^{3} \mathrm{P} ;{ }^{9} \mathrm{G}\end{array}$ & $\begin{array}{l}1.730 \\
1.600\end{array}$ & $\begin{array}{l}1.726 \\
1.592 \\
1.002 \\
1.137 \\
0.901 \\
1.442 \\
\end{array}$ & $\begin{array}{l}-0.004 \\
-0.008\end{array}$ \\
\hline $\begin{array}{r}J=6 \\
4 f^{7} 5 d^{9} \mathrm{D}\end{array}$ & 11128.22 & $\begin{array}{l}11093 \\
38786 \\
40071 \\
40533\end{array}$ & 35 & $\begin{array}{l}97.33 \\
92.38 \\
92.16 \\
89.51\end{array}$ & $\begin{array}{l}{ }^{8} \mathrm{~S} ;{ }^{9} \mathrm{D} \\
{ }^{6} \mathrm{~T} ;{ }^{\mathrm{I}} \\
{ }^{6} \mathrm{I} ;{ }^{5} \mathrm{I} \\
{ }^{7} \mathrm{~F},{ }^{3} \mathrm{~F} ;{ }^{9} \mathrm{I}\end{array}$ & $\begin{array}{l}1.68 \\
3.38 \\
4.14 \\
3.68\end{array}$ & $\begin{array}{l}{ }^{6} \mathrm{P} ;{ }^{7} \mathrm{~F} \\
{ }^{7} \mathrm{~F} ;{ }^{3} \mathrm{~F} ;{ }^{7} \mathrm{I} \\
{ }^{7} \mathrm{~F} ;{ }^{3} \mathrm{~F} ;{ }^{5} \\
{ }^{7} \mathrm{~F},{ }^{1} \mathrm{D} ;{ }^{7} \mathrm{H}\end{array}$ & 1.665 & $\begin{array}{l}1.659 \\
1.144 \\
1.073 \\
1.239\end{array}$ & -0.006 \\
\hline$J=7$ & & $\begin{array}{l}38822 \\
40102 \\
41717\end{array}$ & & $\begin{array}{l}93.03 \\
92.73 \\
91.06\end{array}$ & $\begin{array}{l}{ }^{6} \mathrm{I} ;{ }^{7} \mathrm{I} \\
{ }^{6} \mathrm{I} ;{ }^{5} \mathrm{I} \\
{ }^{7} \mathrm{~F},{ }^{3} \mathrm{~F} ;{ }^{9} \mathrm{I}\end{array}$ & $\begin{array}{l}2.81 \\
3.91 \\
2.59\end{array}$ & $\begin{array}{l}{ }^{7} \mathrm{~F},{ }^{3} \mathrm{~F} ;{ }^{7} \mathrm{I} \\
{ }^{7} \mathrm{~F} ;{ }^{3} \mathrm{~F} ;{ }^{5} \mathrm{I} \\
{ }^{7} \mathrm{~F},{ }^{1} \mathrm{D} ;{ }^{7} \mathrm{H}\end{array}$ & & $\begin{array}{l}1.233 \\
1.179 \\
1.303\end{array}$ & \\
\hline$J=8$ & & $\begin{array}{l}38875 \\
40169 \\
43032\end{array}$ & & $\begin{array}{l}93.56 \\
93.16 \\
92.96\end{array}$ & $\begin{array}{l}{ }^{6} \mathrm{I} ;{ }^{7} \mathrm{I} \\
{ }^{6} \mathrm{I} ;{ }^{5} \mathrm{I} \\
{ }^{7} \mathrm{~F},{ }^{3} \mathrm{~F} ;{ }^{9} \mathrm{I}\end{array}$ & $\begin{array}{l}2.38 \\
3.71 \\
2.45\end{array}$ & $\begin{array}{l}{ }^{7} \mathrm{~F},{ }^{3} \mathrm{~F} ;{ }^{7} \mathrm{I} \\
{ }^{7} \mathrm{~F},{ }^{3} \mathrm{~F} ;{ }^{5} \mathrm{I} \\
{ }^{7} \mathrm{~F},{ }^{3} \mathrm{~F} ;{ }^{7} \mathrm{I}\end{array}$ & & $\begin{array}{l}1.292 \\
1.251 \\
1.346\end{array}$ & \\
\hline$J=9$ & & $\begin{array}{l}38951 \\
44481\end{array}$ & & $\begin{array}{l}94.07 \\
95.69\end{array}$ & $\begin{array}{l}{ }^{6} \mathrm{I} ;{ }^{7} \mathrm{I} \\
{ }^{7} \mathrm{~F},{ }^{3} \mathrm{~F} ;{ }^{9} \mathrm{I}\end{array}$ & $\begin{array}{l}2.04 \\
1.71\end{array}$ & $\begin{array}{l}{ }^{7} \mathrm{~F},{ }^{3} \mathrm{~F} ;{ }^{7} \mathrm{I} \\
{ }^{7} \mathrm{~F},{ }^{3} \mathrm{~F} ;{ }^{7} \mathrm{I}\end{array}$ & & $\begin{array}{l}1.334 \\
1.376\end{array}$ & \\
\hline
\end{tabular}


spin-orbit interaction between configurations differing in quantum number $n$ of one electron (with $l>0)$. The above effects can influence the hyperfine splittings as well. In precise hyperfine structure calculations it is very important to include mixing effect of various electronic states via interaction with the nucleus. This leads to the "breakdown" of $J$ quantum number describing total angular momentum of the electronic shell. Methods of semiempirical calculations of the atomic structure taking into account the effects discussed above have been subject of our studies for many years. As a result many-body parametrization methods of fine- as well as hyperfine-structure in many-configurations-approximation have been elaborated [4, 5], The parametrization methods have been used by us in the analysis of the structure of many atoms e. g. Ti [6],V [7], Co [8], Ta [9], Si [10], Pb [11],

\section{1. Parametrization of matrix elements of Hamiltonian}

The Hamiltonian allowing to describe the fine structure of an atom is written traditionally in the form

$$
H=H_{0}+H_{1}
$$

where

$$
\begin{aligned}
& H_{0}=\sum_{i}\left(\frac{\hat{p}_{i}^{2}}{2 m}-\frac{Z e^{2}}{r_{i}}+U\left(r_{i}\right)\right) \\
& H_{1}=H_{N C}+H_{S O}+H_{M M}
\end{aligned}
$$

and the particular terms stand for as follows:

$$
\begin{array}{ll}
U(r) & - \text { cental part of Coulomb repulsion of electrons, } \\
H_{N C}=\sum_{i>j} \frac{e^{2}}{r_{i j}}-\sum_{i} U\left(r_{i}\right) & - \text { noncentral part of Coulomb repulsion, } \\
H_{S O} & - \text { spin-orbit interaction, } \\
H_{M M} & - \text { so called mixed magnetic interaction. }
\end{array}
$$

The Hamiltonian (4) permits to account for relativistic effects though the operators which constitute (4) are treated as effective operators acting on nonrelativistic wave function. The forms of the operators can be found in the papers [12-16],

It is not possible to evaluate the exact infinite energy matrix of this Hamiltonian; therefore the matrix has been truncated to a finite space, called a model space, containing only strongly interacting configurations.

The influence of distant configurations is included in the energy matrix calculated in the extended model space by addition of second order corrections to the first-order matrix elements

$$
\left\langle\Psi\left|H_{1}\right| \Psi^{\prime}\right\rangle
$$


The corrections are given by:

$$
C\left(\Psi, \Psi^{\prime}\right)=-\sum_{\Psi_{1} \neq \Psi, \Psi^{\prime}} \frac{\left\langle\Psi\left|H_{1}\right| \Psi_{1}\right\rangle\left\langle\Psi_{1}\left|H_{1}\right| \Psi^{\prime}\right\rangle}{\Delta E}
$$

where

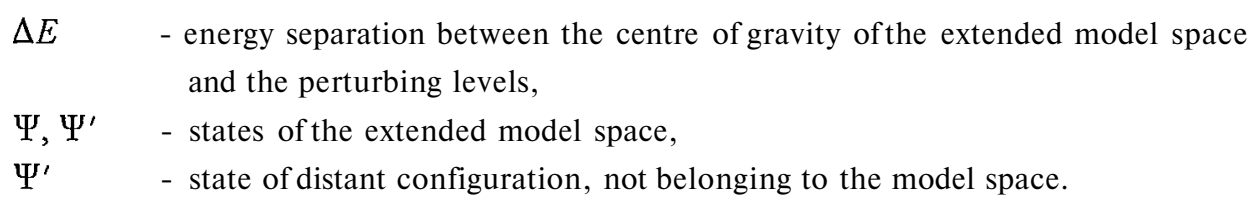

The operator $\mathrm{H}_{1}$ in the formula (5) should be the sum of all the operators occurring in the first-order perturbation theory. However, when calculating the second-order effects in the fine structure analysis it is customaiy to truncate the Hamiltonian to three terms: the central part of the interelectronic interactions $\left(H_{C}\right)$, the noncentral Coulomb repulsion $\left(H_{C R}\right)$ and the spin-orbit interaction $\left(H_{S O}\right)$.

The construction of the energy matrix of the Hamiltonian (4) augmented with the effective operators representing the second-order effects of perturbation theory requires calculation of numerous integrals dependent on the angular co-ordinates and various radial parameters. The integrals over angular co-ordinates may be determined exactly, what is not possible in the case of the radial integrals. Therefore the matrix elements of the Hamiltonian are regarded as linear combinations of radial integrals where the angular integrals serve as the expansion coefficients. The radial integrals are treated as free (or constrained) parameters which can be reckoned fitting the calculated levels to the experimental ones by the least squares method. It is very important to define the radial-integral parameters lucidly, since their starting values for the iterative fs LSQ-fit procedure can be taken from $a b$ initio calculations, and because of their physical interpretation.

\subsection{Hyperfine structure}

The hyperfine structure splitting of atomic levels originates from the interaction of the electrons with the nuclear magnetization and non-spherical nuclear charge distribution. For all except for the most accurate measurements the hyperfine interaction can be described very well by the magnetic-dipole $(M 1)$ and electric-quadrupole $(E 2)$ interaction, and the results expressed in terms of nuclear magnetic-dipole and electric-quadrupole moments. If the hyperfine interaction is treated as a perturbation of the fine structure interaction, the hyperfine energy can be expressed as:

$$
W_{F}(\Psi)=\sum_{\Phi, \Phi^{\prime}} c(\Psi, \Phi) c\left(\Psi, \Phi^{\prime}\right)\left\langle\Phi I F M_{F}\left|H_{h f s}(M 1)+H_{h f s}(E 2)\right| \Phi^{\prime} I F M_{F}\right\rangle
$$

where $\Psi \equiv(S L J)$ denotes the real fs state written in $S L$-basis, $\Phi \equiv(v S L J)$ are the SL-basis wavefunctions for the complete Hamiltonian, $I, F, M_{F}$ are quantum numbers, $c(\Psi, \Phi)$ are eigenvector amplitudes. $H_{h f s}(M 1)$ and $H_{h f s}(E 2)$ are the familiar one-body hfs operators given by Sandars and Beck [17] in the following forms: 


$$
\begin{gathered}
H_{h f s}(M 1)=I \cdot \sum_{n l}\left[a_{n l}^{01} 1-(10)^{1 / 2} a_{n l}^{12}\left(s \times C^{(2)}\right)^{(1)}+a_{n l}^{10} s\right] \\
H_{h f s}(E 2)=T_{n}^{(2)} /(e Q) \sum_{n l}\left[b_{n l}^{02}\{(2 l(l+1)(2 l+1)) /((2 l-1)(2 l+3))\}^{1 / 2} U^{(02) 2}+\right. \\
\left.+(3 / 10)^{1 / 2} b_{n l}^{13} U^{(13) 2}+(3 / 10)^{1 / 2} b_{n l}^{11} U^{(11) 2}\right]
\end{gathered}
$$

where $a_{n l}^{\kappa k}$ and $b_{n l}^{\kappa k}$ are one-electron parameters, which can be treated either as free parameters, or calculated theoretically $a b$ initio. The hyperfine energy can be written in the usual form:

$$
W_{F}(\Psi)=\frac{K}{2} A(\Psi)+\frac{3 K(K+1)-4 I J(I+1)(J+1)}{8 I J(2 J-1)(2 I-1)} B(\Psi)
$$

where

$$
K=F(F+1)-I(I+1)-J(J+1)
$$

and $A(\Psi)$ and are the $h f s$ constants for magnetic-dipole and electric-quadrupole interactions, respectively. Combining (6) and (9) we obtain

$$
\begin{aligned}
& A(\Psi)=\sum_{\Phi, \Phi^{\prime}} c(\Psi, \Phi) c\left(\Psi, \Phi^{\prime}\right) A\left(\Phi, \Phi^{\prime}\right) \\
& B(\Psi)=\sum_{\Phi, \Phi^{\prime}} c(\Psi, \Phi) c\left(\Psi, \Phi^{\prime}\right) B\left(\Phi, \Phi^{\prime}\right)
\end{aligned}
$$

A further factorization of the contributions from single electrons into angular and radial parts in the case of configuration $l^{N} l_{1} l_{2}$ yields:

$$
\begin{aligned}
& A(\Psi)=\sum_{\Phi, \Phi^{\prime}} c(\Psi, \Phi) c\left(\Psi, \Phi^{\prime}\right)\left[\alpha\left(\Phi, \Phi^{\prime}\right)_{n l}^{01} a_{n l}^{01}+\alpha\left(\Phi, \Phi^{\prime}\right)_{n l}^{12} a_{n l}^{12}+\alpha\left(\Phi, \Phi^{\prime}\right)_{n l}^{10} a_{n l}^{10}+\right. \\
& +\alpha\left(\Phi, \Phi^{\prime}\right)_{n_{1} l_{1}}^{01} a_{n_{1} l_{1}}^{01}+\alpha\left(\Phi, \Phi^{\prime}\right)_{n_{1} l_{1}}^{12} a_{n_{1} l_{1}}^{12}+\alpha\left(\Phi, \Phi^{\prime}\right)_{n_{1} l_{1}}^{10} a_{n_{1} l_{1}}^{10}+ \\
& +\alpha\left(\Phi, \Phi^{\prime}\right)_{n_{2} l_{2}}^{01} a_{n_{2} l_{2}}^{01}+\alpha\left(\Phi, \Phi^{\prime}\right)_{n_{2} l_{2}}^{12} a_{n_{2} l_{2}}^{12}+\alpha\left(\Phi, \Phi^{\prime}\right)_{n_{2} l_{2}}^{10} a_{n_{2} l_{2}}^{10} \\
& B(\Psi)=\sum_{\Phi, \Phi^{\prime}} c(\Psi, \Phi) c\left(\Psi, \Phi^{\prime}\right)\left[\beta\left(\Phi, \Phi^{\prime}\right)_{n l}^{02} b_{n l}^{02}+\beta\left(\Phi, \Phi^{\prime}\right)_{n l}^{13} b_{n l}^{13}+\beta\left(\Phi, \Phi^{\prime}\right)_{n l}^{11} b_{n l}^{11}+\right. \\
& +\beta\left(\Phi, \Phi^{\prime}\right)_{n_{1} l_{1}}^{02} b_{n_{1} l_{1}}^{02}+\beta\left(\Phi, \Phi^{\prime}\right)_{n_{1} l_{1}}^{13} b_{n_{1} l_{1}}^{13}+\beta\left(\Phi, \Phi^{\prime}\right)_{n_{1} l_{1}}^{11} b_{n_{1} I_{1}}^{11}+ \\
& \left.+\beta\left(\Phi, \Phi^{\prime}\right)_{n_{2} l_{2}}^{02} b_{n_{2} l_{2}}^{02}+\beta\left(\Phi, \Phi^{\prime}\right)_{n_{2} l_{2}}^{13} b_{n_{2} l_{2}}^{13}+\beta\left(\Phi, \Phi^{\prime}\right)_{n_{2} l_{2}}^{11} b_{n_{2} l_{2}}^{11}\right]
\end{aligned}
$$

are the angular coefficients originating from the one-body $h f s$ operator.

The second-order perturbation theory has been used to calculate the "repulsion effect" between different $h f s$ sublevels with the same quantum number $F$. The second order energy shift is given by

$$
\delta W_{F}(\Psi, J)=\frac{\left|\left\langle\Psi, J I F M_{F}\left|H_{h f s}\right| \Psi^{\prime}, J^{\prime} I F M_{F}\right\rangle\right|^{2}}{E(\Psi, J F)-E\left(\Psi^{\prime}, J^{\prime} F\right)}
$$


where $|\Psi\rangle=\mid \Phi S L>$ denotes the real fine-structure $S L J$ state (wave function in intermediate coupling) written in $S L$ basis and $I, F, M_{F}$ are good quantum numbers characterizing the real $h f S$ state.

\section{THE COMPUTER PACKAGE}

The structure of the computer package prepared and adopted for calculations by the use of vector computers is given in Fig. 5. EMATRIX and ATOM programs described in this paper are the part of a set of FORTRAN programs for fine- and hyperfine structure analysis of complex atoms. These programs can be run on any system provided with a FORTRAN compiler. EMATRIX provides routines for the generation of energy matrix for configurations up to four open shells. This program has modular structure. The main block works as an analyser: takes an input data, verifies conformity with the physical rales and chooses the proper modules to calculate the energy matrix coefficients. The output data from EMATRIX (binary file) constitutes the input for ATOM program.

ATOM program can be used for semiempirical analysis of the fine structure of an atom. At present working versions of EMATRIX and ATOM programs are written using FORTRAN COMPILER with several features supporting the concurrency offered by the computer system. Another important concept, supported by Parallel FORTRAN, is that of a task being multithreaded. The task can contain any number of concurrent processes (threads) running on the same processor. Threads within a task can communicate with each other via shared memoiy. The software construction of semaphores, prevent threads from interfering with each other while operating on shared data. There is a non-trivial problem to parallelize the algorithm for calculation of the energy matrix coeffiecients. First the sequential version has been optimized and a speed up factor of about 2-3 on an average has been obtained. Next step for vectorization and parallelization of our code is to remove data dependency, wherever possible. Because of a large amount of modules this stage is still in progress. As shown in the ATOM program, iteration method for the fine structure analysis is used. In each iteration Householder method of reduction of real symmetric matrix to tridiagonal form is used and eigenvalues and eigenvectors of a symmetric tridiagonal matrix are calculated. Good speed results on the CRAY computer has been obtained when, instead of calling our own procedures, TRED2 (Householder reduction of real symetric matrix) and TQL2 (eigenvalues and eigenvectors of a symetric tridiagonal matrix) from the fully vectorized CRAY SCIENTIFIC LIBRARY ROUTINES have been used.

\section{APPLICATION OF THE COMPUTER PACKAGE TO INTERPRETATION OF EXPERIMENTAL RESULTS OF Eu ${ }^{+}$}

As it was mentioned above, in the interpretation of experimental results for the ground level and low-lying metastable levels of the $4 f^{7} 6 s$ configuration in $\mathrm{Eu}^{+}$the contributions due to the spin-orbit interaction with high-lying levels belonging to the same configuration, as well as the electrostatic interactions with other odd parity configurations, have to be considered. Hence in 


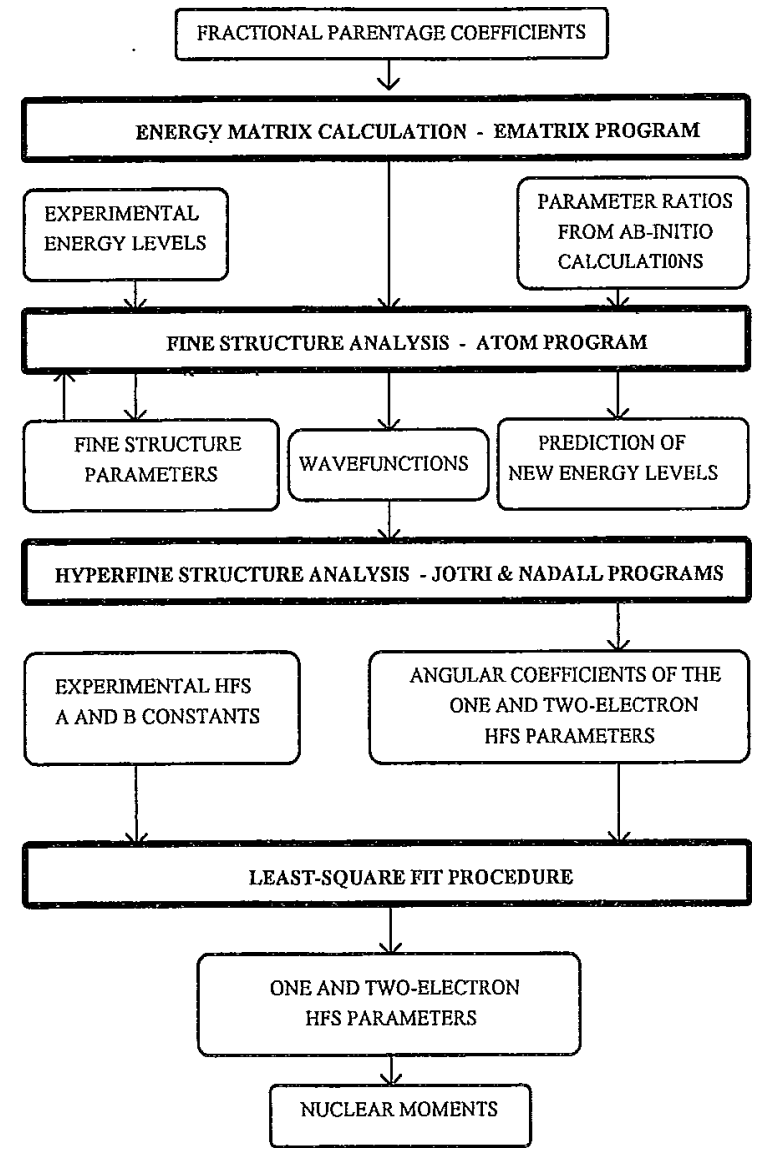

Fig. 5. Scheme of the computer package

the analysis of experimental results the system of 4 configurations interacting each other has been taken into account:

$$
4 f^{7} 6 s+4 f^{7} 5 d+4 f^{6} S d 6 p+4 f^{6} 6 s 6 p
$$

The rank of the generated energy matrix here is 5654, it includes 15 submatrices. The largest one (for $J=4$ ) is of the rank 840 . This means that for the one state with $J=4$ we are able to consider the contributions from 839 other states in the first-order of perturbation theory. In Table I the experimental energies are compared with the calculated ones. Moreover we are able to predict the energy values for the levels non-observed so far. In this table only the energy values of several lowest lying levels of interest are presented as an example. The whole number of the eigenvalues calculated in our procedure was 5653 in the energy region of $172000 \mathrm{~cm}^{-1}\left(51.6 \times 10^{14} \mathrm{~Hz}\right)$. For each eigenvalue the relevant eigenvector has been calculated. For example 10 leading components of linear combination of the eigenvector of the ground state $4 f^{7} 6 s \quad \mathrm{~S}_{4}$ are given: 


$\begin{array}{ll}4 f^{7}\left({ }^{8} \mathrm{~S}\right) 6 s^{9} \mathrm{~S} & 0.973714 \\ 4 f^{6}\left({ }^{7} \mathrm{~F}\right) 5 d 6 p\left({ }^{3} \mathrm{~F}\right){ }^{9} \mathrm{~S} & 0.185617 \\ 4 f^{7}\left({ }^{6} \mathrm{P}\right) 6 s^{7} \mathrm{P} & 0.128966 \\ 4 f^{6}\left({ }^{5} \mathrm{G} 1\right) 5 d 6 p\left({ }^{3} \mathrm{~F}\right){ }^{7} \mathrm{P} & 0.011909 \\ 4 f^{6}\left({ }^{5} \mathrm{D} 1\right) 5 d 6 p\left({ }^{3} \mathrm{~F}\right){ }^{7} \mathrm{P} & 0.011346 \\ 4 f^{6}\left({ }^{7} \mathrm{~F}\right) 5 d 6 p\left({ }^{3} \mathrm{~F}\right){ }^{7} \mathrm{P} & 0.011123 \\ 4 f^{6}\left({ }^{5} \mathrm{G} 3\right) 5 d 6 p\left({ }^{3} \mathrm{~F}\right){ }^{7} \mathrm{P} & 0.010209 \\ 4 f^{6}\left({ }^{5} \mathrm{~F}\right) 5 d 6 p\left({ }^{3} \mathrm{~F}\right){ }^{7} \mathrm{P} & 0.009166 \\ 4 f^{7}\left({ }^{6} \mathrm{D}\right) 6 s{ }^{7} \mathrm{D} & 0.007397 \\ 4 f^{6}\left({ }^{5} \mathrm{D} 3\right) 5 d 6 p\left({ }^{3} \mathrm{~F}\right){ }^{7} \mathrm{P} & 0.007161\end{array}$

It should be mentioned that in the above combination the amplitude of the $\left.44 f\left({ }^{7} \mathrm{~F}\right) 5 d 6 p,{ }^{3} \mathrm{~F} ;{ }^{9} \mathrm{~S}_{4}\right)$ state with expected energy value of $49089 \mathrm{~cm}^{-1}$ is big enough to influence the experimental A $\left(4 f^{7} 6 s^{9} \mathbf{S}_{4}\right)$ constant: the contribution to the A-constant amounts to $3.6 \times 10^{6} \mathrm{~Hz}$ and exceeds the experimental error. It indicates, that also the contributions resulting from the interaction with high lying levels of different configurations should be taken into account if very precise $h f s$ analysis is performed. Such $h f s$ analysis is possible if precise wavefunctions are available from $f s$ calculations with the use of ATOM program (see Fig. 5). The wavefunctions are necessary to generate the angular coefficients using JOTRI and NAD ALL programs. In the least square fit procedure the equations (12) and (13) are resolved and the values of $h f s$ radial parameters are calculated. The values of $h f s$ parameters together with angular coefficients obtained from our calculations allow one to predict the values of $h f s$ constants for the levels not investigated so far. We can give expected values for the $h f s$ constants representing magnetic dipole-, electric quadrupole-, magnetic octupole- and electric hexadecapole interactions, i.e. A, B, C and D constants. They are expressed as functions of $h f s$ radial parameters $a_{n l}^{\mathrm{k} k}, b_{n l}^{\mathrm{\kappa} k}, c_{n l}^{\mathrm{k} k}$ and $d_{n l}^{\mathrm{kk}}$, respectively. Below the $h f_{s}$ constants for the ground state of $\mathrm{Eu}^{+}$are given as an example.

Similarly, a set of parametrical equations can be written for each excited state of $\mathrm{Eu}^{+}$. If the number of experimental constants is large enough to construct a redundant equation system, the

$$
\begin{array}{lll}
\mathrm{A}=\quad & 0.41793198 \times 10^{-2} & a_{4 f}^{01} \\
+ & 0.13022700 \times 10^{-2} & a_{4 f}^{12} \\
+ & 0.87082016 & a_{4 f}^{10} \\
+ & 0.12499992 & a_{6 s}^{10} \\
\mathrm{~B}=\quad & 0.59323538 \times 10^{-3} & b_{4 f}^{02} \\
- & 0.33951466 \times 10^{-4} & b_{4 f}^{13} \\
+ & 0.38021516 \times 10^{-1} & b_{4 f}^{11} \\
\mathrm{C}=\quad & 0.44732952 \times 10^{-4} & c_{4 f}^{03} \\
+ & 0.44984004 \times 10^{-2} & c_{4 f}^{12} \\
& -0.23339774 \times 10^{-6} & d_{4 f}^{04} \\
\mathrm{D}=\quad & 0.34290063 \times 10^{-7} & d_{4 f}^{15} \\
& +0.32122783 \times 10^{-4} & d_{4 f}^{13}
\end{array}
$$


values of $h f s$ radial parameters can be calculated. These values may be applied directly for determination of nuclear moments. At present appropriate experimental works are in progress.

\section{CONCLUSIONS}

In this work the atomic structure analysis of a complex system performed for europium ion has been presented as an example of the extensive semiempirical fine- and hyperfine structure calculations. These calculations have been possible through the use of computer package prepared for vectors computers. The results of the calculations have shown that configuration interactions are significant and they have to be considered in interpretation of very precise measurements in a Paul trap.

\section{References}

[1] H. G. Dehmelt, P. E. Toschek, Bull. Am. Phys. Soc., 20, 60 (1975)

H. G. Dehmelt, Phys. Scr., T22, 102 (1988)

[2] Th. Sauter, R. Blatt, W. Neuhauser, P. E. Toschek, Phys. Scr., T22, 128 (1988)

[3] R. H. Dicke, Phys. Rev., 89, 472 (1953)

[4] J. Dembczyński et al., Phys. Scr., 54, 444 (1996)

[5] J. Dembczyński, W. Ertmer, U. Johann, P. Unkel, Z. Phys., A321, 1 (1985)

[6] E. Stachowska, Z. Phys. D 42, 33 (1997)

[7] P. Palmeri et al., Phys. Scr., 55(5), 585 (1997);

P.Unkel et al., Z. Phys., D11, 259 (1989)

[8] J. Dembczyński, G. H. Guthohrlein, E. Stachowska, Phys. Rev., A48, 4, 2752 (1993)

[9] J. Dembczyński et al., Z.Phys. D 39, 143 (1997)

[10] J. Dembczyński, E. Stachowska, Phys. Scr., 43, 248 (1991)

[11] J. Dembczyński et al., Phys. Rev., A49(2), 745 (1994)

[12] L. Jr. Armstrong, Theory of the Hyperfine Structure of Free Atoms, Wiley-Interscience, New York 1971

[13] I. Lindgren, A. Rosen., Case Stud. At. Phys. 4, 97 (1970)

[14] I. Lindgren, J. Morrison, Atomic Many-Body Theory, Springer, Berlin 1982

[15] L. Jr. Armstrong, S. Feneuille, Adv. Atom. Molec. Phys., 10, 1 (1974)

[16] L. Jr. Armstrong, S. Feneuille, Phys. Rev., 173, 58 (1968)

[17] P. G. H. Sandars, J. Beck, Proc. Roy. Soc., A289, 97 (1965) 